\title{
Combined Relay Selection in Spatially Random Networks
}

\author{
Tianhao Fu, Shuping Dang, Member, IEEE, Linping Wu, and Miaowen Wen, Senior Member, IEEE
}

\begin{abstract}
In deterministic networks where relay nodes are stationary, combined relay selection is an efficient relay selection scheme, which is capable of achieving the near-optimal outage performance with much lower system complexity. However, the efficiency of combined relay selection is only validated upon fixed topological settings. To investigate the performance of combined relay selection in spatially random networks (SRNs), we employ the Poisson point process to model the dynamical nature of cooperative networks and study the outage performance of the proposed system. It is surprising that the equivalence principle of combined relay selection appearing in deterministic networks does not hold in SRNs.
\end{abstract}

Index Terms-Combined relay selection, multi-carrier system, outage performance, and spatially random network (SRN).

\section{INTRODUCTION}

Cooperative communication technologies have been developing rapidly, especially after incorporating paradigms of multi-carrier communications [1]. To harvest spatial diversity, various multi-carrier relay selection schemes were proposed [2]. The most well-known schemes refer to the bulk and per-subcarrier relay selection schemes [3]. The outage and error performance of both schemes is analyzed in [4], [5]. They are also applied in conjunction with various advanced communication techniques [6]-[8]. To maximize the benefits and minimize the drawbacks of both well-known relay selection schemes, another efficient selection scheme termed the combined relay selection is proposed in [9]. By reducing the full set of relays for selection to a subset of only two relays, combined relay selection is of much lower system complexity and can save a considerable amount of signaling overhead for coordination among multiple relays [10]. Meanwhile, it is surprising that by a subtly designed selection algorithm, combined relay selection is found capable of achieving the near-optimal outage performance in the high signal-to-noise ratio (SNR) region. This unique attribute is later called the equivalence principle [11].

Though insightful, all the above works assume static application scenarios, where all relays are placed in a given

This work was supported in part by the National Natural Science Foundation of China under Grant 61871190 and and in part by the Fundamental Research Funds for the Central Universities under Grant 2019SJ02.

T. Fu is with Graduate School of China Academy of Engineering Physics (CAEP), Beijing 100088, China, and Institute of Applied Physics and Computational Mathematics, Beijing 100094, China (e-mail: futianhao18@gscaep.ac.cn).

S. Dang is with Computer, Electrical and Mathematical Science and Engineering Division, King Abdullah University of Science and Technology (KAUST), Thuwal 23955-6900, Saudi Arabia (e-mail: shuping.dang@kaust.edu.sa).

L. Wu is with Institute of Applied Physics and Computational Mathematics, Beijing 100094, China (e-mail: wlp@iapcm.ac.cn).

M. Wen is with the School of Electronic and Information Engineering, South China University of Technology, Guangzhou 510641, China (e-mail: eemwwen@scut.edu.cn). and invariant topology. This static configuration implies a fixed number of relay nodes and unchanged relay locations. As node mobility becomes prevalent in $5 \mathrm{G}$ communication systems, the usefulness of these analyses based on static application scenarios is limited. In this regard, we study a stochastic cooperative communication system with combine relay selection for the decode-and-forward (DF) relaying protocol in SRNs. We, in particular, propose a timer-based hybrid relay selection implementation scheme for SRNs and study the effects of different spatial distribution parameters on the outage performance. Overall, the main contributions of this paper are summarized as follows:

- Different from deterministic topological settings, we introduce combined relay selection to the SRN and tailor the selection procedure to fit its random nature.

- We study the outage performance for combined relay selection in SRNs.

- We simulate the combined relay selection scheme in SRNs and demonstrate that the equivalence principle of combined relay selection is not applicable for SRNs.

\section{SySTEM MODEL}

In the space domain, we postulate a two-dimensional (2D) $\mathrm{SRN}$, in which source and destination nodes are fixed but the relay nodes are randomly distributed over the $2 \mathrm{D}$ polar coordinate system according to homogeneous Poisson point process (PPP) with a constant density $\lambda$. Without loss of generality, the polar coordinates of source, destination, and relay are denoted as $\mathbf{p}_{S}=(0,0), \mathbf{p}_{D}=\left(r_{S D}, 0\right)$, and $\mathbf{p}_{m}=\left(r_{S m}, \theta_{m}\right)$, respectively, where $r_{(\cdot)}$ denotes the distance between two nodes; $\theta_{m}$ denotes the angle of the $m$ th relay with reference to the coordinate origin. The set of a random number of relays is represented by $\mathcal{M}$. Also, we denote $r_{m D}$ as the distance between the $m$ th relay node and the destination, which is a dependent variable of $r_{S m}$ and $\theta_{m}$. Assuming that the network topology does not change throughout the entire transmission process over the transmitting and forwarding phases, $r_{m D}$ can be determined by $r_{S m}$ and $\theta_{m}$ according to the law of cosines: $r_{m D}=\left(r_{S D}^{2}+r_{S m}^{2}-2 r_{S D} r_{S m} \cos \left(\theta_{m}\right)\right)^{\frac{1}{2}}$. Further assume that the shape of the $2 \mathrm{D}$ distribution region of the relay nodes $\mathcal{D}_{\varsigma} \subseteq \mathbb{R}^{2}$ as a disk of radius $\varsigma$ and the center of the disk is located at the source node. Therefore, we have $r_{S m}<\varsigma$ and $\theta_{m} \in[0,2 \pi), \forall m \in \mathcal{M}$.

In the frequency domain, we assume that there are $K$ orthogonal subcarriers when orthogonal frequency division multiplexing (OFDM) is employed for encoding parallel data on multiple subcarrier frequencies. The set of all $K$ subcarriers is represented by $\mathcal{K}$. Over all subcarrier frequencies at all communication nodes, the noise is an additive white Gaussian noise with average power $N_{0}$. All noise terms are independent 
and identically distributed. Two signal attenuation mechanisms, i.e., path loss and multi-path fading are considered, which give the instantaneous SNR at the $m$ th relay on the $k$ th subcarrier as $\operatorname{SNR}_{1}(m, k)=P_{t} G_{1}(m, k) r_{S m}^{-\alpha} / N_{0}$, where $P_{t}$ is the uniform transmit power assigned to all communication nodes; $\alpha$ is the path loss exponent characterizing the severity of the path loss; $G_{i}(m, k)$ is the $i$ th hop channel power gain between the terminal node and the $m$ th relay over the $k$ th subcarrier, which is used to capture the severity of multipath fading. Supposing that the Rayleigh fading model is adopted in this paper, the channel power gain is assumed to be independent and exponentially distributed with unit mean.

By employing the DF relaying protocol at all relays, the instantaneous SNR for the forwarded signal in the second hop can be similarly expressed as $\operatorname{SNR}_{2}(m, k)=$ $P_{t} G_{2}(m, k) r_{m D}^{-\alpha} / N_{0}$. Consequently, the equivalent end-to-end (E2E) SNR incorporating the path loss and multi-path fading over both hops is given by [12]

$$
\operatorname{SNR}(m, k)=\min \left\{\operatorname{SNR}_{1}(m, k), \operatorname{SNR}_{2}(m, k)\right\}
$$

\section{Combined Relay SElEction Scheme IN SRNS}

Given the SRN configurations introduced in the previous section, we detail the combined relay selection criterion in this section. Before going into the details of the combined relay selection scheme, we emphasize that different from deterministic networks, the number of relays and relay locations are highly dynamic in SRNs. For this reason, classic centralized channel estimation and the associated relay selection implementation schemes become impractical, because a plethora of signaling overhead will be generated, which significantly affects the transmission of intended information. To enable the implementation of the combined relay selection scheme in SRNs, we tailor the classic timer-based relay selection implementation scheme [13], and propose a timer-based hybrid relay selection implementation scheme in this paper.

Leveraging this timer-based hybrid scheme, combined relay selection can be implemented in SRNs with much lower system complexity. This is because the signaling overhead of active detection and coordination among multiple relays is reduced. Specifically, in the first distributed stage, we assume that all relays are equipped with $K$ timers. Each timer is associated with each subcarrier by setting an indicator as $T(m, k)=\beta / \min \left\{G_{1}(m, k) r_{S m}^{-\alpha}, G_{2}(m, k) r_{m D}^{-\alpha}\right\}$, where $\beta$ is a preset constant for mitigating the multitimer collision problem, which is also known among all communication nodes. Once the timer reaches the time of $T(m, k)$ after receiving the ready-to-send (RTS) message from the source, the $m$ th relay will broadcast a clear-tosend (CTS) message over the $k$ th subcarrier, which will also be detected by the source. Because $\beta$ is known at the source, the source can easily retrieve the E2E channel quality $Q(m, k)=\min \left\{G_{1}(m, k) r_{S m}^{-\alpha}, G_{2}(m, k) r_{m D}^{-\alpha}\right\}$ by estimating the response time $T(m, k)$. In this way, the source can construct an $M \times K$ matrix $\mathbf{Q}$ of E2E channel quality and use this matrix to carry out centralized relay selection ${ }^{1}$.

To reduce the latency, we can further set threshold time $\tau$ at the source for the first distributed stage, beyond which the broadcast CTS messages are neglected, and an $\tilde{M} \times K$ incomplete sub-matrix $\tilde{\mathbf{Q}}$ can be constructed to facilitate lowcomplexity centralized relay selection at the source, where $\tilde{M} \leq M$ is the number of relays that broadcast on at least one subcarrier within $\tau$ and is thereby a dependent random variable on $M$. In this way, the source is capable of eliminating a large number of relays that are not suited for forwarding information due to disadvantageous locations and thereby reducing the signaling overhead. When setting the threshold time for the timer-based hybrid relay selection implementation scheme, it is worth noting that

- We assume that $M \gg K$ in SRNs, and therefore, the number of columns of $\tilde{\mathbf{Q}}$ remains to be $K$.

- We set the unmeasured E2E channel qualities in $\tilde{\mathbf{Q}}$ to zero.

Knowing $\mathbf{Q}$ or $\tilde{\mathbf{Q}}$ in a decentralized manner, the source can perform combined relay selection on a centralized basis.

To simplify the following analysis and demonstration, we assume that $\tau$ is large enough to make $\mathbf{Q}$ perfectly known by the source. Therefore, the $M \times K$ matrix of E2E SNR can be constructed at the source. Subsequently, combined relay selection can be carried out by two steps: the first step is termed the selection set shrinking, and the second step is simply the per-subcarrier relay selection over the $\eta$ relays in the shrunk selection set $\mathcal{L}_{\eta}^{*}$.

To shrink $\mathcal{M}$ with $M$ relays to a subset of $\eta$ relays, there are $Z=\left(\begin{array}{c}M \\ \eta\end{array}\right)$ possible ways, and each will result in a unique combination of $\eta$ relays, denoted as $\mathcal{L}_{\eta}(z)$, $\forall z \in \mathcal{Z}=\{1,2, \ldots, Z\}$. The optimal subset $\mathcal{L}_{\eta}^{*}$ can be yielded by combined selection by the following criterion:

$$
\mathcal{L}_{\eta}^{*}=\arg \max _{z \in \mathcal{Z}} \min _{k \in \mathcal{K}} \max _{m \in \mathcal{L}_{\eta}(z)}\{\operatorname{SNR}(m, k)\} .
$$

In the second step, the signal sent over the $k$ th subcarrier by the source is forwarded by the optimal relay in $\mathcal{L}_{\eta}^{*}$ according to the following criterion:

$$
m(k)=\arg \max _{m \in \mathcal{L}_{\eta}^{*}}\{\operatorname{SNR}(m, k)\} .
$$

From the above formulations, it is clear that when $\eta=1$ and $\eta=M$, combined selection can be reduced to bulk relay selection and per-subcarrier relay selection, respectively. For clarity, we illustrate the pseudocode of the combined relay selection procedure in Algorithm 1.

\section{Outage Performance Analysis}

In this paper, we employ the system outage probability to capture the reliability performance of combined relay selection in SRNs. Again, different from deterministic networks, the number of spatially distributed relays is random, and thereby the classic definition of system outage event for cooperative

\footnotetext{
${ }^{1}$ For simplicity, we assume in this paper that the time measurement at the source is sufficiently accurate so that the multi-timer collision problem becomes negligible; otherwise the optimization of $\beta$ should be taken into consideration, which is out of the scope of this paper [14].
} 


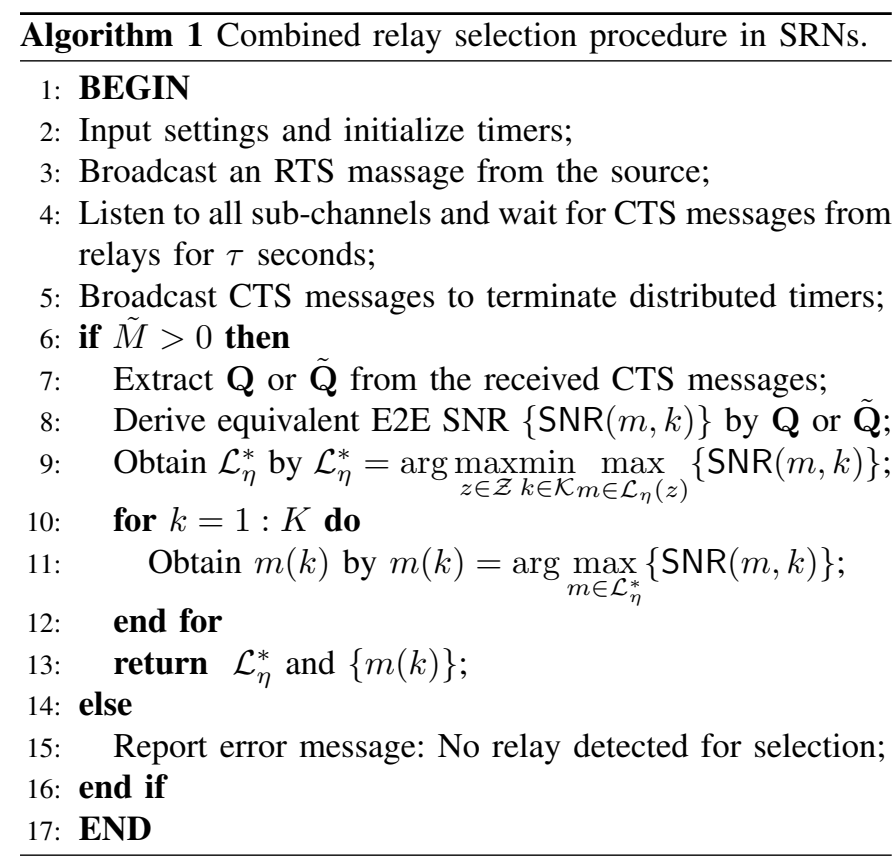

OFDM systems given in [3] needs to be amended. We follow the outage definition proposed in [15] and formulate the system outage probability for cooperative OFDM systems as

$$
\Phi(s)=\mathbb{P}\left\{\left\{\min _{k \in \mathcal{K}} \max _{m \in \mathcal{L}_{\eta}^{*}}\{\operatorname{SNR}(m, k)\}<s\right\} \bigcup\{\mathcal{M}=\varnothing\}\right\},
$$

where $\mathbb{P}\{\cdot\}$ denotes the probability of the random event enclosed, and $s$ is a preset outage threshold of SNR determined by the configurations and accuracy of the receiver at the destination. The amended system outage probability is used as an indicator to evaluate the reliability through simulations. Note that, $\mathcal{M}=\varnothing$ means that the number of relays distributed over $\mathcal{D}_{\varsigma}$ is zero, and there is no relay for selection. We also regard this special case as an outage event.

According to the combined selection criterion, the frequency- and space-domain pairing is carried out in terms of the orders of channel power gains. This will inevitably result in an NP-complete discrete combinatorial optimization problem, which is mathematically intractable. To the best of the authors' knowledge, the only existing tool that can quantitatively analyze the asymptotic outage performance of combined relay selection at high SNR is Theorem 1 proposed in [11]. However, this lemma is proposed upon the assumption of a deterministic relay network and does not work for SRNs by averaging over multiple topological realizations. This is because the number of relay nodes is varying in SRNs. As a result, we can only indirectly estimate the outage performance of combined relay selection in SRNs from its quantitative relation with bulk and per-subcarrier relay selection schemes in deterministic relay networks.

By (2), (3) and (4), we can express the system outage probability of combined relay selection as $\Phi(s)=\underset{\Pi\left(\mathcal{D}_{\varsigma}\right)}{\mathbb{E}}\left\{\Phi\left(s \mid \Pi\left(\mathcal{D}_{\varsigma}\right)\right)\right\}$, where $\Phi\left(s \mid \Pi\left(\mathcal{D}_{\varsigma}\right)\right)=$ $\mathbb{P}\left\{\min _{k \in \mathcal{K}} \max _{m \in \mathcal{L}_{\eta}^{*}}\{\operatorname{SNR}(m, k)\}<s \mid \Pi\left(\mathcal{D}_{\varsigma}\right)\right\} \quad$ is the outage probability of a given topological realization of relay nodes, and $\Pi\left(\mathcal{D}_{\varsigma}\right)$ denotes the homogeneous PPP over disc $\mathcal{D}_{\varsigma}$. Once the topological realization is given, we can derive the following relation among the outage probabilities of combined, bulk, and per-subcarrier relay selection schemes: $\Phi_{\mathrm{ps}}\left(s \mid \Pi\left(\mathcal{D}_{\varsigma}\right)\right) \leq \Phi\left(s \mid \Pi\left(\mathcal{D}_{\varsigma}\right)\right) \leq \Phi_{\text {bulk }}\left(s \mid \Pi\left(\mathcal{D}_{\varsigma}\right)\right)$. Note that, the equality of the above relation holds if and only if $\mathcal{M}=\varnothing$, leading to $\Phi_{\mathrm{ps}}\left(s \mid \Pi\left(\mathcal{D}_{\varsigma}\right)\right)=\Phi\left(s \mid \Pi\left(\mathcal{D}_{\varsigma}\right)\right)=$ $\Phi_{\text {bulk }}\left(s \mid \Pi\left(\mathcal{D}_{\varsigma}\right)\right)=1$. That is, there is no relay for selection, and the two-hop data transmission must be in outage regardless of the adopted relay selection scheme. As the same spatial distribution of relay nodes is applied, we can thereby have the relation among the system outage probabilities averaging over $\Pi\left(\mathcal{D}_{\varsigma}\right)$ to be $\Phi_{\mathrm{ps}}(s)<\Phi(s)<\Phi_{\text {bulk }}(s)$. From this inequality, it is straightforward to have $\Delta(s)=\Phi(s)-\Phi_{\mathrm{ps}}(s)<\Phi_{\text {bulk }}(s)-\Phi_{\mathrm{ps}}(s)$, and $\Delta(s)>0$.

When $P_{t} / N_{0} \rightarrow \infty$, we can refer to the asymptotic outage performance analyses for bulk and per-subcarrier relay selection schemes presented in [15] and obtain

$$
\begin{array}{r}
\Delta(s)<\exp \left(-\lambda \pi \varsigma^{2}\right)\left(K-1+\exp \left(\frac{K \lambda \pi \varsigma^{2} s N_{0} \tau_{\alpha}\left(r_{S D}, \varsigma\right)}{P_{t}}\right)\right. \\
\left.-K \exp \left(\frac{\lambda \pi \varsigma^{2} s N_{0} \tau_{\alpha}\left(r_{S D}, \varsigma\right)}{P_{t}}\right)\right)
\end{array}
$$

where $\tau_{\alpha}\left(r_{S D}, \varsigma\right)$ is a constant dependent on $\alpha, r_{S D}$, and $\varsigma$. By (5), we observe that for a bounded and small distribution radius $\varsigma, \lim _{P_{t} / N_{0} \rightarrow \infty}\left\{\Phi_{\text {bulk }}(s)-\Phi_{\text {ps }}(s)\right\}=0$. Therefore, we have $\lim _{P_{t} / N_{0} \rightarrow \infty}\{\Delta(s)\}=0$ according to the squeeze theorem and obtain the outage floor of combined relay selection in SRNs: $\Phi(s) \sim \exp \left(-\lambda \pi \varsigma^{2}\right)=\mathbb{P}\{\mathcal{M}=\varnothing\}$, leading to a zero-diversity-order cooperative system. However, for a large distribution radius $\varsigma, \lim _{P_{t} / N_{0} \rightarrow \infty}\left\{\Phi_{\text {bulk }}(s)-\Phi_{\mathrm{ps}}(s)\right\}$ becomes an indeterminate form. Consequently, without the exact knowledge of $\Phi(s)$, it would not be possible to get the insight into the asymptotic outage performance of combined relay selection in SRNs from an analytical perspective.

\section{Numerical Evaluation AND CONClusion}

To investigate the outage performance of combined relay selection in SRNs from a numerical perspective, we numerically evaluate the outage performance and investigate the impacts of several key network parameters as follows. We normalize $s=1$ and let $\eta=2$ in all simulations so as to reduce the computational burden at the source, which is aligned with the classic configurations of the combined selection criterion originated in [11]. We also suppose that $\mathbf{Q}$ is perfectly known at the source and destination. First of all, we simulated the relation between outage probability and the ratio of transmit power to noise power $P_{t} / N_{0}$ in Fig. 1 , in which we set $\lambda=1$ and $\varsigma=5$. From this figure, we can clearly observe several key attributes of combined relay selection over SRNs. Comparing combined relay selection with per-subcarrier selection, the all-important observation is that the equivalence principle of combined relay selection presenting in deterministic networks does not hold anymore for SRNs, as there is an obvious gap between the outage performance of combined relay selection and per-subcarrier relay selection. Also, this gap remains 


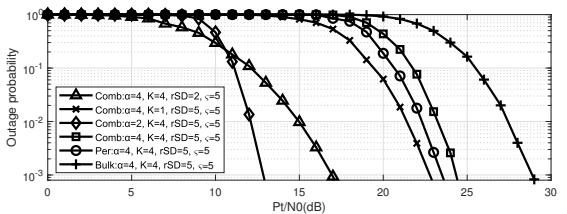

Fig. 1: Outage probability versus $P_{t} / N_{0}$ for different multi-carrier relay selection schemes in SRNs, given $\eta=2, K \in\{1,4\}, \alpha \in$ $\{2,4\}, r_{S D} \in\{2,5\}, \lambda=1$, and $\varsigma=5$.

constant in the high SNR region and will not be closed by increasing $P_{t} / N_{0}$. On the other hand, compared to the performance of bulk relay selection, combined relay selection still has a significant performance gain by simply adding one more relay in the shrunk selection set (i.e., $\eta=2$ ). This indicates that the combined relay selection scheme is still able to improve the reliability of cooperative OFDM systems when relay nodes are randomly distributed, albeit losing the optimality in the asymptotic region. Considering the trade-off between reliability and system complexity, the usefulness and feasibility of the combined relay selection scheme for SRNs can be corroborated.

By observing Fig. 1, we can also obtain the insights into the effects of $K, \alpha$ and $r_{S D}$ on the outage performance of combined relay selection in SRNs. Specifically, with a reduced number of subcarriers $K$, the probability that the worst subcarrier, i.e., the one with the lowest E2E SNR, is in outage is lower. This implies better outage performance. Also, if the terminal distance $r_{S D}$ is shrunken, the average propagation distances over the first and the second hops are reduced, which can lead to better outage performance, as expected. Moreover, the path loss exponent $\alpha$ dominates the outage performance over other parameters, and lowering $\alpha$ yields a considerable improvement on the outage performance.

We also simulated the relations between outage probability and relay distribution density $\lambda$ as well as relay distribution radius $\varsigma$ in Fig. 2 and Fig. 3, respectively. From Fig. 2, it is evident that increasing the relay distribution density is an efficient way to enhance the system reliability when relay nodes are randomly distributed, because, on the average, there are more relay nodes for selection, which will thereby yield a higher probability to find high-quality E2E channels. These results comply with the basic principle of the superdense network paradigm. The results illustrated in Fig. 3 are also aligned with our expectation, because in most cases, the appropriate relay nodes that can successfully forward the signals from the source to the destination are located in the middle area between terminal nodes. Consequently, increasing the relay distribution radius will only help when the radius is small. Once the distribution radius goes far larger than the distance between terminal nodes $r_{S D}$, further increasing the radius will only yield a negligible performance improvement.

In conclusion, the most important observation from the above numerical results is that the equivalence principle of combined relay selection appearing in deterministic networks does not hold in SRNs. Hence, combined relay selection can only be regarded as a compromise between outage performance and system complexity in SRNs. By increasing the

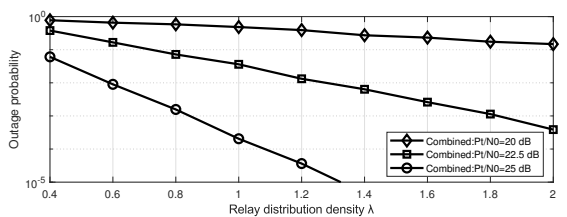

Fig. 2: Outage probability versus relay distribution density $\lambda$ in SRNs, given $\eta=2, K=4, \alpha=4, r_{S D}=5$, and $\varsigma=5$.

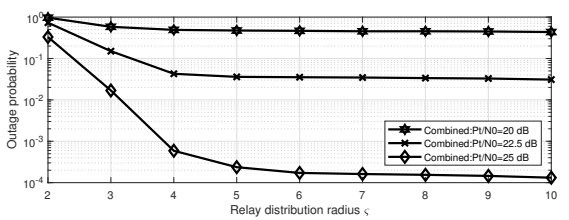

Fig. 3: Outage probability versus relay distribution radius $\varsigma$ in SRNs, given $\eta=2, K=4, \alpha=4, r_{S D}=5$, and $\lambda=1$.

number of activated relays, combined selection scheme trades off higher system complexity for better outage performance.

\section{REFERENCES}

[1] M. Pejanovic-Djurisic, E. Kocan, and R. Prasad, OFDM Based Relay Systems for Future Wireless Communications, ser. River Publishers Series in Communications Series. River Publishers, 2012.

[2] B. Gui, L. Dai, and L. J. Cimini Jr., "Selective relaying in cooperative OFDM systems: Two-hop random network," in Proc. IEEE WCNC, Las Vegas, NV, USA, Mar. 2008, pp. 996-1001.

[3] W. Yang and Y. Cai, "On the performance of the block-based selective OFDM decode-and-forward relaying scheme for 4G mobile communication systems," Journal of Communications and Networks, vol. 13, no. 1, pp. 56-62, Feb. 2011.

[4] W. Yang, W. Yang, and Y. Cai, "Outage performance of OFDM-based selective decode-and-forward cooperative networks over Nakagami-m fading channels," Wireless Personal Communications, vol. 56, no. 3, pp. 503-515, 2011.

[5] Q. Zhang, F. Shu, M. Wang, and J. Sun, "Relay selection schemes for precoded cooperative OFDM and their achievable diversity orders," IEEE Signal Process. Lett., vol. 18, no. 4, pp. 231-234, 2011.

[6] O. Amin and M. Uysal, "Adaptive power loading for multi-relay OFDM regenerative networks with relay selection," IEEE Trans. on Commun., vol. 60, no. 3, pp. 614-619, 2012.

[7] M. Banar and A. Mohammadi, "Characterisation of relay selection in cooperative multiple-input multiple-output-orthogonal frequency division multiplexing systems," IET Comm., vol. 8, no. 18, pp. 3290-3297, 2014.

[8] S. Dang, G. Chen, and J. P. Coon, "Multicarrier relay selection for full-duplex relay-assisted OFDM D2D systems," IEEE Trans. on Veh. Technol., vol. 67, no. 8, pp. 7204-7218, Aug. 2018.

[9] Y. Li, W. Wang, and F. Zheng, "Combined bulk and per-tone relay selection in cooperative OFDM systems," in Proc. IEEE ICCC, Beijing, China, Aug. 2012, pp. 487-491.

[10] Q. Huang, M. Ghogho, J. Wei, and P. Ciblat, "Practical timing and frequency synchronization for OFDM-based cooperative systems," IEEE Trans. on Signal Process., vol. 58, no. 7, pp. 3706-3716, July 2010.

[11] S. Dang, J. P. Coon, and G. Chen, "An equivalence principle for OFDMbased combined bulk/per-subcarrier relay selection over equally spatially correlated channels," IEEE Trans. on Veh. Technol., vol. 66, no. 1, pp. 122-133, Jan. 2017.

[12] T. Q. Duong, V.N.Q. Bao, and H. Zepernick, "On the performance of selection decode-and-forward relay networks over Nakagami-m fading channels," IEEE Commun. Lett., vol. 13, no. 3, pp. 172-174, Mar. 2009.

[13] A. Bletsas, A. Khisti, D. P. Reed, and A. Lippman, "A simple cooperative diversity method based on network path selection," IEEE J. Sel. Area. Comm., vol. 24, no. 3, pp. 659-672, Mar. 2006.

[14] W. Song, P. Ju, A. Jin, and Y. Cheng, "Distributed opportunistic two-hop relaying with backoff-based contention among spatially random relays," IEEE Trans. on Veh. Technol., vol. 64, no. 5, pp. 2023-2036, May 2015.

[15] S. Dang, J. P. Coon, and G. Chen, "Outage performance of two-hop OFDM systems with spatially random decode-and-forward relays," IEEE Access, vol. 5, pp. 27 514-27 524, 2017. 